\title{
Does screening and brief intervention for drug use in primary care increase receipt of substance use disorder treatment?
}

\author{
Richard Saitz ${ }^{1 *}$, Theresa Kim² ${ }^{2}$ Judith Bernstein ${ }^{2}$, Debbie M Cheng ${ }^{3}$, Jeffrey Samet ${ }^{4}$, Christine Lloyd-Travaglini ${ }^{5}$, \\ Tibor Palfai ${ }^{6}$, Jacqueline German ${ }^{2}$ \\ From INEBRIA 12th Congress, \\ Atlanta, GA, USA. 24-25 September 2015
}

\section{Background}

Little is known about the efficacy of "RT" (referral to treatment) for increasing receipt of substance use disorder (SUD) treatment by patients with unhealthy drug use identified by screening. We compared receipt of SUD treatment between baseline and 6 months across three randomized groups: no intervention and two different types of brief interventions.

\section{Material and methods}

Adults presenting to a hospital-based primary care clinic with recent drug use (Alcohol, Smoking and Substance Involvement Screening Test [ASSIST] drug specific scores of $i 4$ ) were enrolled in a randomized clinical trial comparing: (1) a 10-15 minute structured interview conducted by health educators (BNI), (2) a 30-45 minute intervention based on motivational interviewing by Masters-level counselors (MOTIV), or 3) no brief intervention. All received information on treatment resources. We assessed receipt of any SUD treatment in a statewide database. Logistic regression analyses adjusted for main drug (self-identified), drug dependence, and past SUD treatment.

\section{Results}

Among 528 participants the main drug was marijuana (63\%), cocaine $(19 \%)$, and opioids $(17 \%)$; $46 \%$ met 12 month drug dependence criteria (Composite International Diagnostic Interview Short Form); 18\% had ASSIST scores $(i 27)$ consistent with dependence (past

\footnotetext{
* Correspondence: rsaitz@bu.edu

${ }^{1}$ Community Health Sciences and General Internal Medicine, Boston University Schools of Public Health \& Medicine/Boston Medical Center, Boston, USA

Full list of author information is available at the end of the article
}

3-months). At 6 months, 14\% (73/528) received any SUD treatment. There were no significant differences in SUD treatment receipt: BNI vs control (adusted odds ratio $[\mathrm{AOR}] 1.16,95 \%$ Confidence Interval $[\mathrm{CI}] 0.59$, 2.30, Hochberg adjusted $\mathrm{p}$-value $=0.66$ ); MOTIV vs control (AOR 0.45, 95\%CI: 0.21, 0.97, Hochberg adjusted $\mathrm{p}$-value $=0.08)$. There were no significant interactions between intervention and main drug, severity (ASSIST), or prior SUD treatment.

\section{Conclusions}

Brief intervention did not increase receipt of SUD treatment in primary care patients. Future research should address how to make referral to treatment successful among screen-identified patients who could benefit from it.

\begin{abstract}
Authors' details
${ }^{1}$ Community Health Sciences and General Internal Medicine, Boston University Schools of Public Health \& Medicine/Boston Medical Center, Boston, USA. ${ }^{2}$ Community Health Sciences, Boston University Schools of Public Health \& Medicine/Boston Medical Center, Boston, USA. ${ }^{3}$ Biostatistics and General Internal Medicine, Boston University Schools of Public Health \& Medicine/Boston Medical Center, Boston, USA. ${ }^{4}$ General Internal Medicine and Community Health Sciences, Boston University Schools of Public Health \& Medicine/Boston Medical Center, Boston, USA. ${ }^{5}$ Data Coordinating Center, Boston University School of Public Health, Boston, USA. ${ }^{6}$ Psychology and
\end{abstract} Brain Sciences, Boston University College of Arts and Sciences, Boston, USA.

Published: 24 September 2015

doi:10.1186/1940-0640-10-S2-046

Cite this article as: Saitz et al:: Does screening and brief intervention for drug use in primary care increase receipt of substance use disorder treatment? Addiction Science \& Clinical Practice 2015 10(Suppl 2):O46.
C Biomed Central

C) 2015 Saitz et al This is an Open Access article distributed under the terms of the Creative Commons Attribution License (http:// creativecommons.org/licenses/by/4.0), which permits unrestricted use, distribution, and reproduction in any medium, provided the original work is properly cited. The Creative Commons Public Domain Dedication waiver (http://creativecommons.org/publicdomain/ zero/1.0/) applies to the data made available in this article, unless otherwise stated. 